\title{
Evaluation of Semisolid Agar Method for Antifungal Susceptibility Test of T.Rubrum.
}

\author{
Sultana Razia', Shaheda Anwar ${ }^{2}$, Md. Ruhul Amin Miah ${ }^{2}$, Najmun Nahar ${ }^{3}$, Ripon Barua ${ }^{4}$ \\ ${ }^{2}$ Department of Microbiology and Immunology, BSMMU, Dhaka
}

\begin{abstract}
:
Background: With increasing fungal disease many newer antifungal drugs are available with different spectrum of activity. Antifungal susceptibility test will help clinicians for selection of effective drug and thereby treatment of patient. Objective: The study was undertaken to perform a simple screening drug susceptibility test of T. rubrum by Semi Solid Agar Antifungal Susceptibility (SAAS) Method. Performance of susceptibility method was assessed by comparing the MICs of three commonly prescribed antifungal agents namely- fluconazole (FCZ), itraconazole (ITZ) and terbinafine (TER) to the CLSI (Clinical and Laboratory Standard Institute) recommended M-38, a broth microdilution method. Results: In SAAS method, among twenty nine T. rubrum, twenty five $(86.2 \%)$ were susceptible (MIC range $0.5-64$ $\mu \mathrm{g} / \mathrm{ml}$ ) to Fluconazole (FCZ) and four (13.7\%) were resistant (MIC value $>64 \mu \mathrm{g} / \mathrm{ml}$ ). In broth microdilution method, among twenty nine T. rubrum, twenty six (89.6\%) were susceptible (MIC range $0.3-64 \mu \mathrm{g} / \mathrm{ml})$ to $\mathrm{FCZ}$ and three (10.3\%) were resistant $(\mathrm{MIC}$ value $>64 \mu \mathrm{g} / \mathrm{ml}$ ). In case of both ITZ and TER, all were susceptible (MIC range $0.3-64 \mu \mathrm{g} / \mathrm{ml}$ ) to both methods. The SAAS method demonstrated the susceptibility pattern of T. rubrum against FCZ, ITZ and TER usually within 72 to 96 hours after organism isolation and results were concordance with the results of CLSI broth microdilution method. Conclusion: Though it is a newer method with proper standardization of the test method, SAAS method is simple and easily applicable screening method for susceptibility testing of antifungal agents against dermatophytes in any microbiology laboratories.
\end{abstract}

Key word: SAAS method, antifungal susceptibility test, dermatophytes.

[BSMMU J 2014; 7 (1) : 11-14]

\section{Introduction:}

Although treatment options are now expanded for fungal pathogen, dermatophytes are also showing resistance to the currently available antifungal drugs like fluconazoles 123. The clinical and laboratory standard institute (CLSI) established reproducible standard method M38-A for antifungal susceptibility test of filamentous fungi which is either broth micro or macro dilution method ${ }^{4}$. Many laboratories do not routinely perform antifungal susceptibility test for many reasons including requirements of special equipments, media and buffering reagent (filter sterilizer, spectophotometric inoculums determination based on conidial size, microtiter tray, RPMI media and MOPS) which are not available for all microbiological

Address for Correspondence: Dr. Sultana Razia

Research Assistant Department of Microbiology \& Immunology BSMMU, Dhaka. Mobile: 01732771303 laboratories in our country ${ }^{5}$. Though it is suitable as reference standard methods, but some wahat the test is laborious, time consuming, expensive, cumbersome and need special expertise to perform ${ }^{56}$. Even today there is no standard, reproducible widely applicable antifungal susceptibility method and has not yet established in medical mycology for dermatophytes ${ }^{3}{ }^{10}$. SAAS (Semi Solid Agar Antifungal Susceptibility) methods have emerged as alternate methods for drug susceptibility test of fungus (yeast and mold) in different laboratories ${ }^{568}$. This method uses inoculums prepared from colony growth and the media is $0.5 \% \mathrm{BHI}$ agar, which is easily available without need of any special equipment or expertise for any microbiological laboratory. Usually the results can be achieved within 72 to 96 hours after initial isolation of fungus. As SAAS screening test should promising result in comparison to CLSI broth microdilution test, may be a useful 
screening method ${ }^{8}$. This study was aimed to introduce SAAS as a simple, relatively quick, cost effective screening method in determining antifungal susceptibility of clinical isolate of dermatophyste.

\section{Methods:}

Test isolates A total of twenty nine T.rubrum strains was isolated from clinical specimen like skin and nail and were studied to assess the applicability of SAAS method for screening of antifungal drug susceptibility test. These were cultured in Sabouraud dextrose agar (SDA) and Dermatophyte test medium (DTM). The cultures were maintained in sterile distilled water at room temperature. For antifungal susceptibility test, the strains were subcultured on potato dextrose agar (PDA) at $28^{\circ} \mathrm{C}$ for 7 days to ensure the viability and purity of the inoculum. Ethical clearance was taken from Institutional Review Board of BSMMU.

Antifungal agents: Three antifungal agents were studied. These includes- flucanozole (FCZ), itraconazole (ITZ) and terbinafine (TER), and were obtained (Square Pharmaceuticals, Bangladesh) in the form of dry powder with known potencies (100\%). FCZ was dissolved in sterile distilled water and ITZ and TER were dissolved in dimethyl sulfoxide (DMSO). Antifungal working solution was prepared according to manufacturers direction as described for the CLSI method. Dilutions were maded in sterile distilled water and same diluents were used to make two fold dilutions of FCZ: ( 0.125 to $64 \mu \mathrm{g} / \mathrm{ml})$, ITZ: ( 0.03 to $64 \mu \mathrm{g} / \mathrm{ml})$ and TER: $(0.03$ to $16 \mu \mathrm{g} / \mathrm{ml})$.

Antifungal susceptibility testing Test procedure of SAAS method: Preparation of antifungal drug supplemented media Five milliliter aliquots of semisolid agar (BHI broth contains $0.5 \%$ agar base) at $\mathrm{pH}$ of 7.4 were prepared in glass tubes under sterile condition. After cooling at $50^{\circ}$ $\mathrm{C}$ in a water bath specific concentration of antifungal working solution was added to the media and kept at 45 to $50^{\circ} \mathrm{C}$ to achieve the final concentration of drugs, eg, FCZ: $(0.125$ to $64 \mu \mathrm{g} / \mathrm{ml})$, ITZ: $(0.03$ to $64 \mu \mathrm{g} / \mathrm{ml})$ and
TER: ( 0.03 to $16 \mu \mathrm{g} / \mathrm{ml})$. Drug free media were prepared to use for growth control (positive control). After cooling, both the drug-supplemented and drug free media containing tubes were stored in sealed plastic bags at $4-8^{\circ} \mathrm{C}$.

Inoculums preparation and incubation: T. rubrum were grown in PDA media at $25^{\circ} \mathrm{C}$ for 4 days and were covered with 4 to $5 \mathrm{ml}$ sterile normal saline and gently rubbed by sterile cotton swab stick soaked with Tween 80 . Then the suspension were transferred to a sterile tube. The suspensions were vortexed. The heavy particles were allowed to settle and the homogenous suspension was adjusted to achieve a tubidity of 0.5 McFarland standard. The semisolid agar media containing specific concentration of antifungal agents (FCZ, ITZ and TER) as well as drugfree controls were prepared in duplicate. Both the media were inoculated with a standard platinum loopful $(0.001$ $\mathrm{ml}$ ) of inoculums suspension by inserting the loop deep within the semisolid agar. A loopful of the onoculum suspension were streaked on to SDA to check for purity and viability of isolated dermatophytes. The tubes were incubated at $35^{\circ} \mathrm{C}$ for 4 days.

Determination of in vitro antifungal susceptibility: MIC of the antifungal agents (FCZ, ITZ and TER) were determined when good growth of dermatiphytes was observed in the drug free medium. The growth in all tubes were compared with that of drug free control CLSI guidelines M38-A and growth was scored in the following way 4: growth comparable to that of drug free control 3: slight decrease in growth (growth approximately $75 \%$ of that of control)

2: significant reduction in growth (growth approximately $50 \%$ of that of control)

1: slight growth or few visible hyphal fragments (growth approximately $25 \%$ of that of control) $)^{0}$ : no visible growth CLSI recommended broth microdilution method was performed in accordance with CLSI-M-38 document ${ }^{4}$. MIC was defined as the lowest concentration that produced complete inhibition of growth (approximately $80 \%$ in azole and $100 \%$ in Terbinafine) ${ }^{3}$. 


\section{Results:}

\section{Table-I}

MICs of three antifungal agents (FCZ, ITZ and TER) in SAAS method against twenty nine T. rubrum.

\begin{tabular}{|c|c|c|c|c|}
\hline $\begin{array}{l}\text { Name of } \\
\text { antifungal } \\
\text { agents }\end{array}$ & $\begin{array}{c}\text { MIC range } \\
\mu \mathrm{g} / \mathrm{ml}\end{array}$ & $\begin{array}{c}\text { Number of } \\
\text { isolates } \\
\mathrm{N}(\%)\end{array}$ & $\begin{array}{l}\text { MIC-50 } \\
(\mu \mathrm{g} / \mathrm{ml})\end{array}$ & $\begin{array}{r}\text { MIC-90 } \\
(\mu \mathrm{g} / \mathrm{ml})\end{array}$ \\
\hline \multirow[t]{3}{*}{$\mathrm{FCZ}$} & $0.5-16$ & $16(55.1)$ & 16 & $>64$ \\
\hline & $32-64$ & $9(31.0)$ & & \\
\hline & $>64$ & $4(13.7)$ & & \\
\hline \multirow[t]{3}{*}{ ITZ } & $0.03-0.5$ & $15(51.7)$ & 0.5 & 8 \\
\hline & $1-4$ & $8(27.5)$ & & \\
\hline & $8-16$ & $6(20.6)$ & & \\
\hline \multirow[t]{3}{*}{ TER } & $0.03-0.5$ & $16(55.1)$ & 0.5 & 8 \\
\hline & $1-4$ & $8(27.5)$ & & \\
\hline & $8-16$ & $5(17.2)$ & & \\
\hline
\end{tabular}

In this study, MICs of three antifungal agents namelyfluconazole (FCZ), itraconazole (ITZ) and terbinafine (TER) demonstrated the susceptibility pattern of twenty nine clinical isolates of $\mathrm{T}$. rubrum by SAAS method (Table 1). In SAAS method for FCZ, among twenty nine T. rubrum, twenty five $(86.2 \%)$ were susceptible (MIC range $0.5-64 \mu \mathrm{g} / \mathrm{ml}$ ) to Fluconazole $(\mathrm{FCZ})$ and four $(13.7 \%$ ) were resistant (MIC value $>64 \mu \mathrm{g} / \mathrm{ml}$ ) $($ Table-1). In case of both ITZ and TER, all isolates were within the MIC range $0.3-64 \mu \mathrm{g} / \mathrm{ml}$ in SAAS method (Table -1). In broth microdilution method, among twenty nine $\mathrm{T}$. rubrum, twenty six $(89.6 \%$ ) were susceptible (MIC range $0.3-64 \mu \mathrm{g} / \mathrm{ml})$ to $\mathrm{FCZ}$ and three $(10.3 \%)$ were resistant (MIC value $>64 \mu \mathrm{g} / \mathrm{ml}$ ). In case of both ITZ and TER all isolates were susceptible (MIC range $0.3-64 \mu \mathrm{g} / \mathrm{ml}$ ) to broth microdilution method. Although the MIC range of both ITZ and TER were same but TER was more susceptible than ITZ against T.rubrum. Table - I also showed MIC50 $(\mu \mathrm{g} / \mathrm{ml})$ and MIC90 $(\mu \mathrm{g} / \mathrm{ml})$ of three antifungal agents (FCZ, ITZ and TER ) 16, 0.5, 0.5 and $>64,8,8$ in SAAS method respectively where $50 \%$ (MIC 50 ) and 90\% (MIC90) T. rubrum were inhibited at specific drug concentration by each antifungal agent.

\section{Discussion:}

Numerous factors have contributed to increase fungal diseases like increasing number of immunosuppressive disease and use of immunosuppressive drugs that enhance interest to do susceptibility test against antifungal drugs ${ }^{911} 12$. There is a need to develop a rapid, reproducible and suitable antifungal susceptibility method which can be performed in any microbiological laboratory. In this study antifungal drugs (FCZ, ITZ and TER) susceptibility test were performed against twenty nine $\mathrm{T}$. rubrum isolates and results were compared with those obtained by CSLI reference broth microdilution method. This study showed among twenty nine T. rubrum isolates in SAAS method, twenty five isolates were susceptible (within recommended MIC range) and four isolates were resistant (out of recommended MIC range) for FCZ (Table I). In broth microdilution method, twenty six isolates were isolates were susceptible (within recommended MIC range) and three isolates were resistant (out of recommended MIC range) for FCZ. In both ITZ and TER all more susceptible (low MIC value) in both methods. This study also determined $\mathrm{MIC}_{50}$ and $\mathrm{MIC}_{90}$ of FCZ, ITZ and TER in both methods and showed TER and ITZ was more effective than FCZ. However TER and ITZ showed low MIC value and more susceptible against dermatophytes than $\mathrm{FCZ}$ which is supported by many studies ${ }^{12313}$. In consideration of MIC range the result of all isolates were within same MIC range except one for FCZ in both methods. Although in both SAAS and broth microdilution methods the antifungal agents (FCZ, ITZ and TER) showed almost same MIC range but their particular MIC value for each isolate in both methods was not same. In SAAS method the MIC value of each isolate had two or more dilutions higher than MIC value in broth microdilution method. This variation may due to high density inoculums preparation causing failure of dermatophyte growth inhibition in SAAS method comparison to quantitative inoculum preparation in CSLI method. Other few study on SAAS method and CSLI recommended standard method also provide comparable data for susceptibility test for antifungal agents ${ }^{568}$. Some variation between two methods may be present even though the practically applied CLSI reference both broth macro and micro dilution methods ${ }^{2}$ 
assess the reproducibility and accuracy of test result, MIC of ATCC strain was not compared.

As SAAS method uses chemically undefined medium and an inoculums of conidia is not standard to conidial size of isolates. So the test is developed as a screening antifungal susceptibility test and as such is not meant to determine MICs or to replace CLSI reference method. Yet to test its validity, MIC comparisons were made with the CLSI test results. For the antifungal agents tested, the concordance of results (within 1 dilution) between the two methods was high. These results suggest that the SAAS method accurately compares to the CLSI method for prediction of susceptibility or resistance of the organism to the drug tested. In future SAAS testing of one drug concentration at the proposed cut - off for resistance would further simplify this screening test and could be considered once MIC endpoints for all drugs are determined for the filamentous fungi. This study compared MIC results of the SAAS screening method and CLSI M- 38 broth microdilution method for three antifungal agents against 29 clinical isolates of dermatophytes. Khan et al., India (2006) determined MIC of dermatophytes in SAAS method and showed $100 \%$ correlation with reference method. Preliminary susceptibility test and identification of isolates can be carried out simultaneously. So further study should be done for susceptibility testing of large number of antifungal agents against yeasts and molds to establish SAAS method, which is simple, cheap, accurate, less time consuming, require no special equipment or expertise and can be performed in any microbiological laboratories.

\section{Conclusion:}

Validation of the SAAS method as an antifungal susceptibility screen requires the correlation of results with the CLSI reference method. Further studies on more antifungal drugs to see the antifungal susceptibility against clinical isolates of yeast and molds should be conducted to establish the SAAS method.

\section{References:}

1. Nweze E.I, Ogbonna CC, Okafor J. (2007) In vitro susceptibility testing of dermatophytes isolated from pediatric cases in Nigeria against five antifungals. Rev. Inst. Med trop. S. Paulo 49(5): 293-295.

2. Pakshir K, Bahaedinie L, Razaei Z, Sodaife M, Zomorodian K. (2009) In vitro activity of six antifungal drugs against clinically important dermatophytes. Jundishapur Journal of Microbiology;2(4): 158-163.

3. Araujo CR, Miranda KC, farnandes ODFL, soares AJ, Silva MDRR. (2009) In vitro susceptibility testing of dermatophytes isolated in Goina, Brazil, against five antifungal agents by broth microdilution method. Rev. Inst. Med. Trop. S. Paulo;51(1):9-12.

4. Espinel-Ingroff A and Canton E. (2007) Antifungal Susceptibility Testing of Filamentous Fungi. Antimicrobial Susceptibility Testing Protocols. 1st edition. Schwalbe R, Steel-Moore and Goodwin AC (eds), CRC press, London. 209-242.

5. Khan S, Singhal S, Mathur T, Upadhyay, Rattan A. (2006) Antifungal susceptibility testing method for resource constrained laboratories. Indian Journal of Medical Microbiology; 24 (3): 171-176.

6. Kuzucu C, Rapino B, McDermott L, and Hadley S. (2004) Comparison of the Semisolid Agar Antifungal Susceptibility Test with the NCCLS M38-P Broth Microdilution Test for Screening of Filamentous Fungi. Journal of Clinical Microbiology; Vol 42 (3): 1224-1227.

7. Serrano MC. (2004) A comparative study of the disc diffusion method with the Broth Microdilution and E-test methods for voriconazole susceptibility testing of Aspergillus spp.J Antimicrob Chemother; $53: 739-42$.

8. Provine H, and Hadley S. (2000) Preliminary Evaluation of a Semisolid Agar Antifungal Susceptibility Test for Yeasts and Molds. Journal of Clinical Microbiology; 38(2): 537-541.

9. Conti s, fanti F, Bertolotti D, Dieci E, Arseni S, Salati A, Polonelli L. Personalized antifungal susceptibility testing. Journal of Antimicrobial Chemitherapy (1999) 43, 333-338.

10. Minas G, carlos Av.A (2006) In vitro susceptibility testing of trichophyton spp. Micological Research; 110: 1355-60.

11. Aal AMA, Taha MM, Mashad NE, Shabrrawy WE. (2007) Antifungal susceptibility testing: New trends. Egyptian Dermatology Online journal; 3(1): 1-10.

12. Jessup CJ, warner J, Islam N, Hasan I, Ghannoum MA. Antifungal susceptibility testing of Dermatophytes: Establishing a medium for inducing conidial growth and evaluation of susceptibility of clinical isolates. J Clin Microbial; 38910: 341-344.

13. Rahim R. (2011) Dermatophytes causing skin, nail and hair infections and sensitivity pattern of Trichophyton rubrum against common antifungal drugs. M.Phil. Thesis. Bangabandhu Sheikh Mujib medical university, Dhaka. 